\title{
Reducing Refractive Index Variations in Compression Molded Lenses by Annealing
}

\author{
Bo Tao", Lianguan Shen ${ }^{1}$, Allen $\mathrm{Yi}^{2}$, Mujun $\mathrm{Li}^{1}$, Jian Zhou ${ }^{1}$ \\ ${ }^{1}$ Department of Precision Machinery and Precision Instrumentation, University of Science and Technology of China, \\ Hefei, Anhui, China \\ ${ }^{2}$ Department of Integrated Systems Engineering, the Ohio State University, Columbus, Ohio, USA \\ Email: taoboq@mail.ustc.edu.cn
}

Received 2013

\begin{abstract}
Compression molding of glass optics is gradually becoming a viable fabrication technique for high precision optical lenses. However, refractive index variation was observed in compression molded glass lenses, which would contribute to image quality degradation. In this research, annealing experiments were applied to control the refractive index variation in molded glass lenses. The refractive index variations pre and post annealing experiment in molded lenses were measured by an experiment setup based on Mach-Zehnder interferometer. The experimental results showed that the refractive index variation can be controlled providing that a proper cooling process is applied during cooling.
\end{abstract}

Keywords: Refractive Index; Mach-Zehnder Interferometer; Optical; Annealing; Compression Molding

\section{Introduction}

Compression molding is a thermal forming process for precision glass optics $[1,2]$. However, refractive index variation was induced in glass during cooling when the glass material went through its glass transition region [3-5]. On the other hand, the variation of refractive index in a molded glass lens will introduce distortion to the wave-front passing through the glass lens, which leads to image quality degradation. In order to ensure a proper optical performance of thermally formed glass lens, it is important to reduce the degree of refractive index variation in the molded glass lens.

Annealing has been studied for improving the quality of glass [6-9]. Through annealing, glass can achieve homogeneous refractive index. Annealing of the compression molding of glass lenses was investigated in this research. In order to identify the effects of annealing on refractive index variations, computer tomography method was employed to measure the refractive index distribution in the glass lenses. The measurement was conducted by using an optical setup based on Mach-Zehnder interferometer $[4,10]$. By comparing experimental results of the glass lens pre and post annealing, the results showed that refractive index variation was reduced.

\section{Design of Experiments}

Glass lenses studied in this research were molded in a commercial glass molding machine (GMP-211V). Ther- mal forming was carried out at $684{ }^{\circ} \mathrm{C}$. After forming, the temperature was maintained until stresses in glass caused by pressing were completely released. Two steps cooling process was followed: the glass lens was cooled to $520{ }^{\circ} \mathrm{C}$ at a rate of $0.8{ }^{\circ} \mathrm{C} / \mathrm{s}$ and then to $200{ }^{\circ} \mathrm{C}$ at a rate of $1.6{ }^{\circ} \mathrm{C} / \mathrm{s}$. When the glass lens was cooled to 200 ${ }^{\circ} \mathrm{C}$, the lens was taken out of the molding machine and cooled naturally at room temperature. Figure $\mathbf{1}$ is the illustration of the glass lens. BK7 was chosen as the glass material. The properties of BK7 are shown in Table 1.

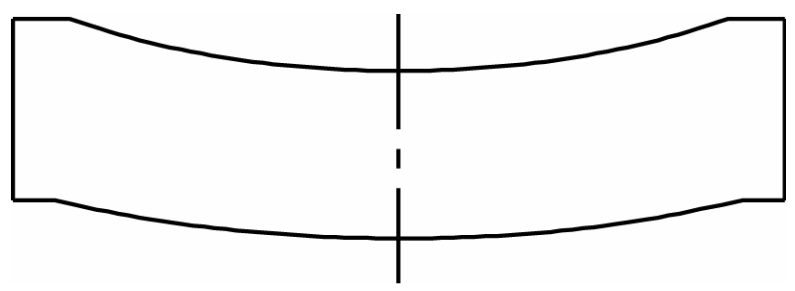

Figure 1. Illustration of the compression molded glass lens.

Table 1. Properties of BK7 glass [11].

\begin{tabular}{ll}
\hline Material Properties & BK7 \\
\hline Elastic modulus, $E[\mathrm{MPa}]$ & 82,500 \\
Poisson's ratio, $v$ & 0.206 \\
Transition temperature, $T_{\mathrm{g}}\left[{ }^{\circ} \mathrm{C}\right]$ & 557 \\
Refractive index, $n$ & 1.5148 \\
\hline
\end{tabular}




\subsection{Refractive Index Measurement}

Figure 2 shows a schematic of the experiment setup used to measure the refractive index in glass lenses. To avoid refraction, tested lens was placed in a box filled with refractive index matching liquid of BK7. He-Ne laser was used as the light source. In the experiment setup, beam splitter 1 divides the laser beam into two. One is used as reference beam, the other one goes through the lens under test. These two beams interfere with each other at beam splitter 2. Fringe pattern carried with refractive index distribution in the lens was captured by a CCD camera.

Figure 3 is the fringe pattern of the molded glass lens at one direction. The fringe pattern was analyzed by a 2D Fourier transform technique [12] and a least-square phase unwrapping method [13] for unwrapped phase. Because of the axisymmetric property of the glass lens, it is sufficient to reconstruct the refractive index distribution in the glass lens through only one fringe pattern. With the unwrapped phase, 3 dimensional (3D) refractive index distribution of the glass lens relative to the refractive index of matching liquid can be reconstructed using the filtered back-projection method [14].

$$
n(x, y, z)=\frac{p(x, y, z) \lambda}{2 \pi d}
$$

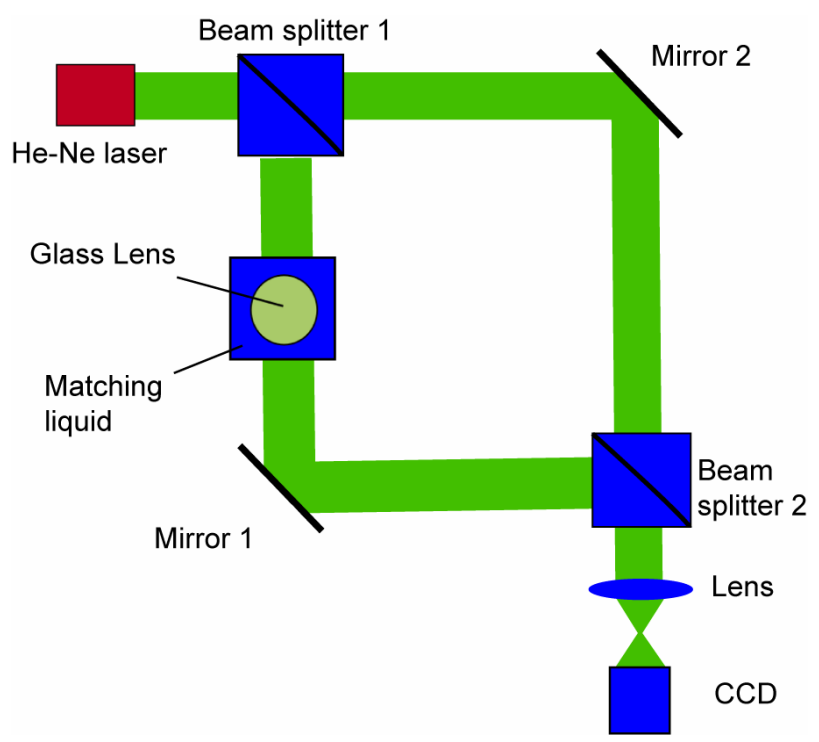

Figure 2. Schematic of the experiment setup for refractive index measurement.

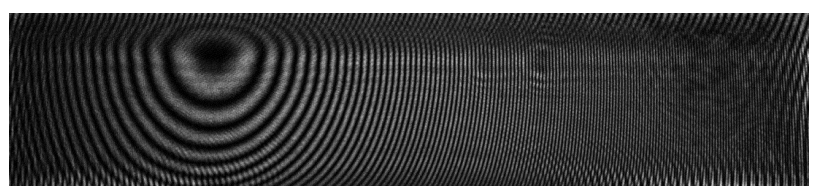

Figure 3. Fringe pattern of the compression molded glass lens before annealing at one direction. where, $p(x, y, z)$ is the reconstructed phase distribution at point $(x, y, z), d$ is the pixel size in the test lens of the interferogram. The reconstructed refractive index in the middle section of the compression molded lens relative to the matching liquid is shown in Figure 4.

\subsection{Annealing}

Annealing experiments were conducted in a commercial furnace (Grieve, BF-12128-HT). Figure 5 illustrates the time-temperature history of the annealing experiments.

At first, the molded glass lens was heated to $560{ }^{\circ} \mathrm{C}$, slightly higher than the glass's transition temperature, and soaked for 10 minutes. After soaking, the glass lens was cooled to $500{ }^{\circ} \mathrm{C}$ at a rate of $1{ }^{\circ} \mathrm{C} / \mathrm{min}$. Then the furnace was turned off. The maximum cooling rate was about $1.29{ }^{\circ} \mathrm{C} / \mathrm{min}$ after turning off the furnace, because there was no force cooling system. Once the temperature decreased to $150{ }^{\circ} \mathrm{C}$, the glass lens was taken out of the furnace and cooled to room temperature naturally. In the experiments, the glass lens under thermal treatment was placed on a ceramic plate in the furnace with the concave side facing down.

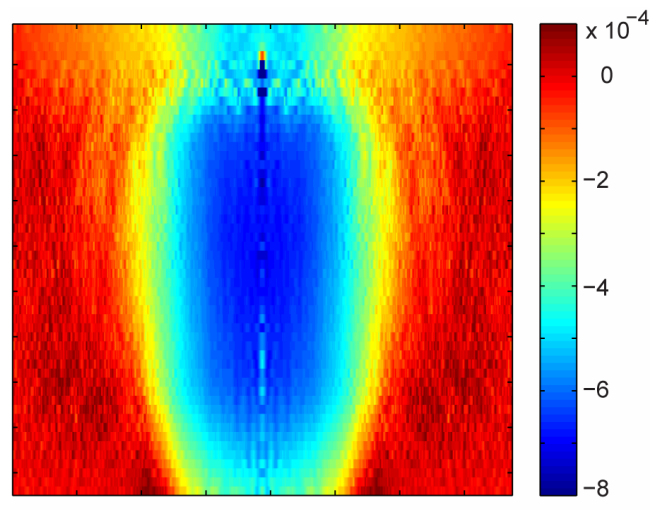

Figure 4. Refractive index distribution of middle section of the molded glass lens relative to the matching liquid.

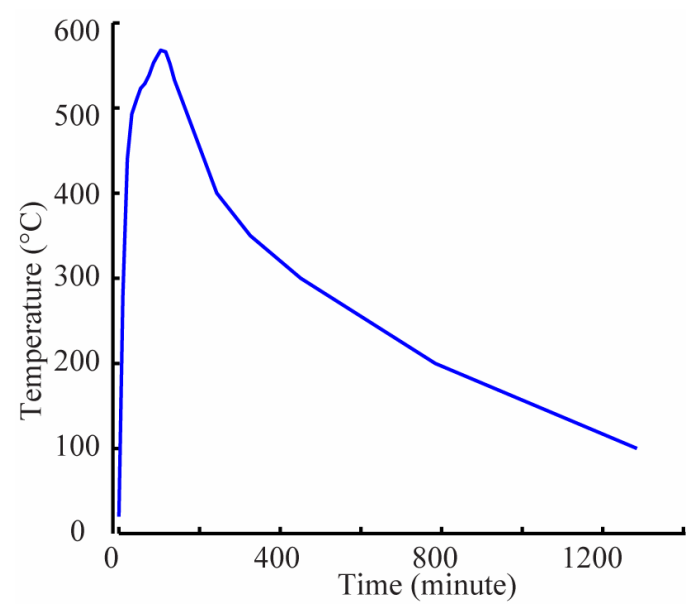

Figure 5. Time-temperature history of annealing. 


\section{Results and Discussion}

Refractive index variations of the molded glass lens pre and post annealing were both measured. Figure 6 shows the average refractive index variations in the middle section of the glass lens along radial direction pre and post annealing experiment. The maximum refractive index variation was reduced about $4 \times 10^{-4}$ which was more than half of the maximum variation before annealing.

The relations between refractive index $n$ and density $\rho$ of the glass material can be described by Lorentz-Lorenz equation [15]:

$$
\frac{n^{2}-1}{n^{2}+2}=\frac{4 \pi}{3} \frac{N_{A} \rho \alpha}{M}
$$

where $N_{A}$ is the Avogadro number, $\alpha$ is the mean polarization and $M$ is the molar weight. Differentiating Equation (2), the relations between refractive index change $d n$ and density change $d \rho$ can be obtained by:

$$
\frac{d n}{d \rho}=\frac{\left(n^{2}-1\right)\left(n^{2}+2\right)}{6 n \rho}
$$

Substituting volume for the density, refractive index change $\Delta n$ can be calculated from the volume change $\Delta V$ and the original volume $V_{\mathrm{o}}$ :

$$
\Delta n=\frac{\left(n^{2}-1\right)\left(n^{2}+2\right)}{6 n}\left(\frac{-\Delta V}{V_{0}+\Delta V}\right)
$$

After annealing, residual stresses inside the glass lens were released. Values of the coefficient of thermal expansion (CTE) of the glass lens were different at different cooling rates due to the behavior of structural relaxation when the glass went through its glass transition region. As such, the volume change was imported by both stresses relaxation and the changes of CTE [16]:

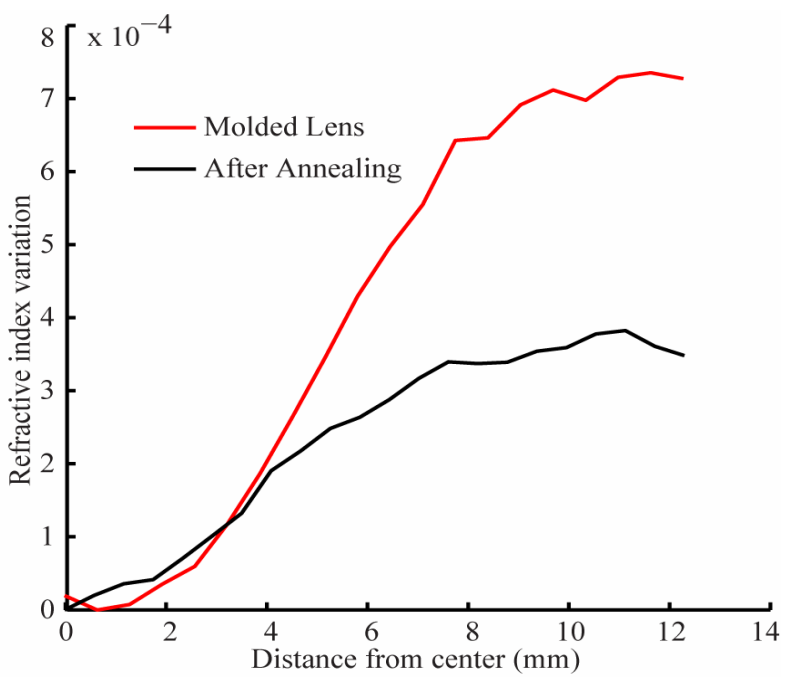

Figure 6. Comparison of the refractive index variations in a molded glass lens pre and post annealing.

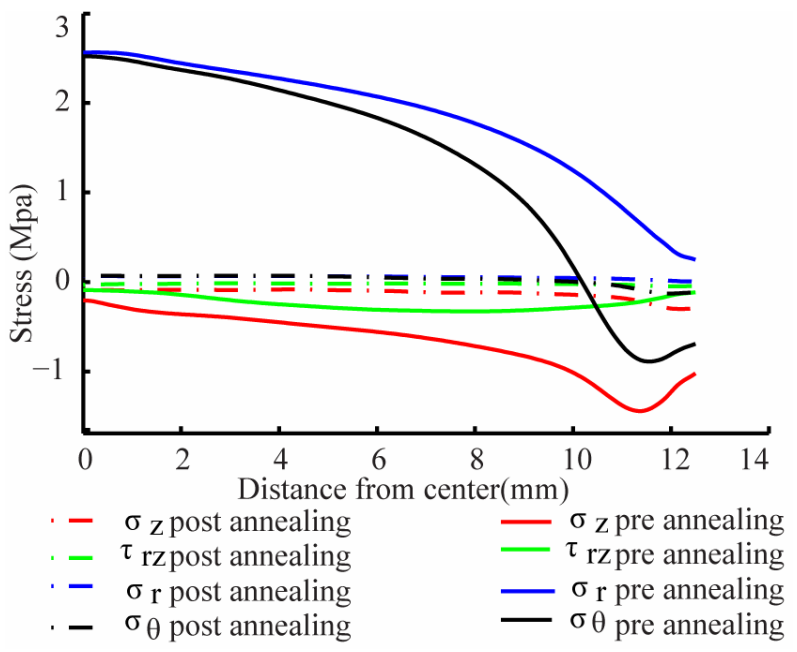

Figure 7. Residual stresses in the glass lens pre and post annealing in cylindrical coordinate.

$$
\begin{aligned}
\Delta V & =V_{o}\left[3 \beta_{h}-3 \beta_{c}\right] \\
& +V_{o}\left[\frac{1-2 v}{E}\left(\sigma_{11}+\sigma_{22}+\sigma_{33}\right)\right]
\end{aligned}
$$

where, $\quad \beta_{h}=\int_{T_{1}}^{T_{2}} \alpha_{h}(T) d T$ and $\beta_{c}=\int_{T_{2}}^{T_{1}} \alpha_{c}(T) d T$.

$\alpha_{h}$ and $\alpha_{c}$ are CTE during heating and cooling, respectively. $T_{1}$ is room temperature and $T_{2}$ is soaking temperature. $v$ is Poisson's ratio and $E$ is elastic modulus. $\sigma_{11}$, $\sigma_{22}$ and $\sigma_{33}$ are the changes of normal stresses along the axes.

The residual stresses inside the glass lens can be measured by a circular polariscope based on the property of birefringence when the glass is stressed [17-19]. The maximum residual stress in the compression molded glass lens was about $3 \mathrm{Mpa}$ in cylindrical coordinate system. Figure 7 shows residual stresses in the middle section of the glass lens pre and post annealing. The residual stresses were significantly released after annealing. Substituting the maximum changes of residual stresses, $E$ and $v$ of the glass lens into Equation (5), the volume change induced by stress relaxation can be calculated, $\Delta V$ $=6 \times 10^{-5} V_{0}$. The maximum refractive index change induced by stresses relaxation was $4 \times 10^{-5}$.

Therefore the refractive index change was mainly caused by the changes of CTE. Basically, slower cooling rates yield lower volumes. In order to get lower refractive index variation, lower cooling rate should be applied.

\section{Conclusions}

Refractive index variations in compression molded glass lenses were investigated using an experiment setup. The refractive index variation was induced during the cooling due to structural relaxation of the glass material. Conse- 
quently the refractive index variation in the glass lens can induce wave-front distortion. To control the refractive index variation, annealing of the glass lenses were conducted and the results demonstrated that the refractive index variations were significantly reduced. This research demonstrated that CTE played a critical role in process optimization for precision compression molded glass optics.

\section{Acknowledgements}

The material is partially based on work supported by National Science Foundation under Grants No. CMMI 0547311. Any opinions, findings, and conclusions or recommendations expressed in this material are those of the authors and do not necessarily reflect the views of the National Science Foundation. The work is also supported by National Natural Science Foundation of China (No. 51075381). Bo Tao acknowledges the financial support from China Scholarship Council.

\section{REFERENCES}

[1] R. O. Maschmeyer, C. A. Andrysick, T. W. Geyer, H. E. Meissner, C. J. Parker and L. M. Sanford, "Precision Molded-Glass Optics," Applied Optics, Vol. 22, No. 16, 1983, pp. 2413-2415. doi:10.1364/AO.22.002413

[2] A. Y. Yi and A. Jain, "Compression Molding of Aspherical Glass Lenses - A Combined Experimental and Numerical Analysis," Journal of the American Ceramic Society, Vol. 88, No. 3, 2005, pp. 579-586. doi:10.1111/j.1551-2916.2005.00137.x

[3] U. Fotheringham, A. Baltes, P. Fischer, P. Höhn, R. Jedamzik, C. Schenk, C. Stolz and G. Westenberger, "Refractive Index Drop Observed After Precision Molding of Optical Elements: A Quantitative Understanding Based on the Tool- Narayanaswamy -Moynihan model," Journal of the American Ceramic Society, Vol. 91, No. 3, 2008, pp. 780-783.doi:10.1111/j.1551-2916.2007.02238.x

[4] W. Zhao, Y. Chen, L. G. Shen and A. Y. Yi, "Investigation of the Refractive Index Distribution in Precision Compression Glass Molding by Use of 3D Tomography," Measurement Science and Technology, Vol. 20, No. 5, 2009, p. 055109. doi:10.1088/0957-0233/20/5/055109

[5] L. J. Su, Y. Chen, A. Y. Yi, F. Klocke and G. Pongs, "Refractive Index Variation in Compression Molding of Precision Glass Optical Components," Applied Optics, Vol. 47 No. 10, 2008, pp. 1662-1667. doi:10.1364/AO.47.001662

[6] J. Wolfe and R. Chipman, "Reducing Symmetric Polarization Aberrations in A Lens by Annealing," Optics Express, Vol. 12, No. 15, 2004, pp. 3443-3451. doi:10.1364/OPEX.12.003443

[7] H. R. Lillie and H. N. Ritland, "Fine Annealing of Optical
Glass," Journal of the American Ceramic Society, Vol. 37, No. 10, 1954, pp. 466-473.

doi:10.1111/j.1151-2916.1954.tb13978.x

[8] H. E. Hagy, "Fine Annealing of Optical Glass for Low Residual Stress and Refractive Index Homogeneity," Applied Optics, Vol. 7, No. 5, 1968, pp. 833-835. doi:10.1364/AO.7.000833

[9] N. M. Brandt, "Annealing of 517.645 Borosilicate Optical Glass: I, Refractive Index," Journal of the American Ceramic Society, Vol. 34, No. 11, 1951, pp. 332-338. doi:10.1111/j.1151-2916.1951.tb13480.X

[10] W. Zhao, Y. Chen, L. G. Shen and A. Y. Yi, "Refractive index and Dispersion Variation in Precision Optical Glass Molding by Computed Tomography," Applied Optics, Vol. 48, No. 9, 2009, pp. 3588-3595. doi:10.1364/AO.48.003588

[11] Schott Glass Inc., Products and Applications, www. schott. de

[12] M. Takeda, H. Ina and S. Kobayashi, "Fourier- Transform Method of Fringe-Pattern Analysis for Computer-Based Topography and Interferometry," Journal of the Optical Society of America, Vol. 72, No. 1, 1982, pp. 156-160. doi:10.1364/JOSA.72.000156

[13] D. C. Ghiglia and L. A. Romero, "Robust Two-Dimensional Weighted and Unweighted Phase Unwrapping That Uses Fast Transforms and Iterative Methods," Journal of the Optical Society of America, Vol. 11, No. 1, 1994, pp. 107-117. doi:10.1364/JOSAA.11.000107

[14] G. T. Herman, "Fundamentals of Computerized Tomography: Image Reconstruction from Projections,' Springer, London 2009. doi:10.1007/978-1-84628-723-7

[15] H. N. Ritland, "Relation between Refractive Index and Density of A Glass at Constant Temperature," Journal of the American Ceramic Society, Vol. 38, No. 2, 1955, pp. 86-88. doi:10.1111/j.1151-2916.1955.tb14581.X

[16] L. J. Su and A. Y. Yi, "Investigation of the Effect of Coefficient of Thermal Expansion on Prediction of Refractive Index of Thermally Formed Glass Lenses Using FEM Simulation," Journal of Non-Crystalline Solids, Vol. 357, No. 15, 2011, pp. 3006-3012. doi:10.1016/j.jnoncrysol.2011.04.005

[17] Anton, A. Errapart, H. Aben and L. Ainola, "A Discrete Algorithm of Integrated Photoelasticity for Axisymmetric Problems," Experimental Mechanics, Vol. 48, No. 5, 2008, pp. 613-620. doi:10.1007/s11340-008-9121-9

[18] A. Errapart, H. Aben, L. Ainola and J. Anton, "Photoelastic Tomography for the Measurement of Thermal and Residual Stresses in Glass," 9th International Congress on Thermal Stresses, Budapest, Hungary, 2011.

[19] H. Aben and A. Errapart, "A Non-Linear Algorithm of Photoelastic Tomography for the Axisymmetric Problem," Experimental Mechanics, Vol. 47, No. 6, 2007, pp. 821-830. doi:10.1007/s11340-007-9057-5 